\title{
Consumo e Conversão Alimentar de Machos Bovinos Inteiros Charolês, Caracu e Cruzamentos Recíprocos em Confinamento ${ }^{1}$
}

\author{
Daniel Perotto², José Luiz Moletta ${ }^{3}$, João Everaldo Prestes de Oliveira ${ }^{4}$, Carlos Lesskiu ${ }^{4}$
}

RESUMO - O consumo diário de matéria seca (CPUPV), a conversão alimentar (CONV) e o ganho de peso médio diário (GMD) de 80 machos bovinos inteiros Charolês (Ch), Caracu (Ca) e seus cruzamentos recíprocos (ChCa e CaCh) gerados pelo cruzamento alternado $\mathrm{Ch}$ x Ca foram analisados. Todos os animais foram alimentados em baias individuais por períodos de 76 a 97 dias, quando receberam silagem de milho à vontade e concentrado contendo 17,8\% PB e 79\% NDT fornecido à base de 1\% PV/animal/dia. As três características foram analisadas inicialmente em função do período de alimentação, sendo o período total dividido em três sub-períodos e, posteriormente, no período total. As médias gerais para as três características, considerando-se o período total, foram 2,25 kg de MS/ $100 \mathrm{~kg}$ PV/dia, 6,75 kg MS/kg de GMD e 1,587 kg/dia, respectivamente, para CPUPV, CONV e GMD. Na análise por período, o grupo genético (GPO) influenciou CPUPV e GMD, mas não a CONV. A interação GPO x período não mostrou efeito para qualquer das três características. O Ch superou o Ca para CONV (-0,861 kg de MS/kg de GMD) e GMD (0,163 kg/dia). A diferença recíproca (ChCa - $\mathrm{CaCh}$ ) foi significativa para as três características, e o ChCa foi melhor que o CaCh. Houve efeito da heterose sobre o GMD. O cruzamento alternado $\mathrm{Ch} \times \mathrm{Ca}$ igualou-se ao $\mathrm{Ch}$ e superou o $\mathrm{Ca}$.

Palavras-chave: bovinos de corte, ingestão de matéria seca, conversão alimentar

\section{Feed Intake and Feed:Gain Ratio of Charolais, Caracu and Reciprocal Crossbred Males in Finished in Feedlot}

\begin{abstract}
Daily dry matter intake (CPUPV), feed:gain ratio (CONV) and average daily gain (ADG) of $80 \mathrm{Charolais}$ (Ch), Caracu (Ca) and reciprocal crossbred $(\mathrm{ChCa}$ and $\mathrm{CaCh}$ ) males were analyzed. The $\mathrm{ChCa}$ and $\mathrm{CaCh}$ were generated by rotational $\mathrm{Ch} \mathrm{x} \mathrm{Ca}$ crossbreeding. All animals were fed in individual stalls for 76 to 97 day periods receiving corn silage ad libitum and a concentrate containing $17.8 \% \mathrm{CP}$ and $79 \% \mathrm{TDN}$ fed $1 \% \mathrm{LW} /$ animal $\bullet$ day. The three traits were analyzed first as a function of period, when the total period was divided into three sub-periods, and, later, in the total period. General means for CPUPV, CONV and ADG were, respectively, 2.25 $\mathrm{kg} \mathrm{DM} / 100 \mathrm{~kg} \mathrm{LW} /$ day, $6.75 \mathrm{~kg} \mathrm{DM} / \mathrm{kg}$ ADG and $1.587 \mathrm{~kg} /$ day. In the analysis by period, the genetic group (GPO) influenced all traits, except for CONV. GPO x period interaction was not significant for any of the three traits. The Charolais was better than the Ca for CONV (-0.861 kg of DM was $/ \mathrm{kg}$ of ADG) and for ADG (0.163 kg/day). The difference (ChCa - CaCh) was significant for the three traits, and the $\mathrm{ChCa}$ was better than its reciprocal. The effect of heterosis was significant for ADG only. Rotational Ch $\mathrm{x} C \mathrm{Ca}$ crossbreeding was equal to Charolais and superior than Caracu.
\end{abstract}

Key Words: beef cattle, dry matter intake, feed:gain ratio

\section{Introdução}

Diferenças genéticas em características relacionadas à eficiência de utilização de alimentos para produção de carne bovina podem se expressar de várias maneiras. Ampla revisão sobre o assunto é encontrada em WARWICK e COBB (1975).

Uma importante fonte de diferenças entre animais quanto à eficiência de produção está na capacidade de ingestão de alimentos, em níveis acima das exigências de manutenção, durante a fase de cres- cimento (LEDGER et al., 1970; GONÇALVES et al., 1991; e OLIVEIRA et al., 1994).

Igualmente importantes para a determinação da eficiência de produção de carne bovina são as diferenças entre animais e entre raças para a taxa de conversão do alimento em peso vivo ou em componentes da carcaça (ARS-USDA, 1974).

As diferenças geralmente observadas em quantidade de alimento consumido por quilograma de ganho de peso ou de carcaça produzidos dependem de fatores como tipo do alimento, temperatura e outras

\footnotetext{
1 Projeto financiado pelo IAPAR/Programa PPA.

2 Pesquisador do IAPAR, Caixa Postal 2031, CEP 80001-979, Curitiba-PR, Endereçco eletrônico: dperotto@pr.gov.br

3 Pesquisador do IAPAR, Caixa Postal 129, CEP 84001-970, Ponta Grossa-PR. Endereço eletrônico: efmiapar@pr.gov.br

${ }^{4}$ Acadêmico do Curso de Agronomia da UEPG e bolsista do PIBIC/CNPq.
} 
variáveis ambientais, peso médio durante o período observado, composição do ganho, estado sanitário, entre outros (GARRETT, 1980). Geralmente, animais de raças mais pesadas, que apresentam maior velocidade de ganho de peso na fase de crescimento, demandam menos alimento por quilograma de peso ganho que animais de raças menores, quando avaliados em faixas de idade e de peso comparáveis (MASON, 1971). Estudos conduzidos nos Estados Unidos da América, comparando raças britânicas com raças européias continentais de grande porte ou com mestiços dessas com as britânicas, foram consistentes em demonstrar que as raças continentais e seus cruzamentos são mais eficientes ganhadores de peso que as britânicas, quando as comparações ocorrem durante um período fixo de tempo Agricultural Research Service - ARS-USDA (1974). Em todos esses estudos, os animais mais eficientes tinham menos gordura na carcaça ao final do período de confinamento.

Os efeitos da heterose sobre a eficiência alimentar geralmente foram pequenos (1\%) e sem significância estatística (LONG, 1980). Entretanto, as estimativas de heterose podem depender da diversidade genética entre as raças envolvidas no cruzamento, bem como do nível alimentar em que os animais experimentais foram mantidos.

O desenvolvimento da bovinocultura de corte no Centro-Sul do Estado do Paraná aponta para modernização crescente, que se caracteriza pela adoção de práticas de manejo reprodutivo, como desmame precoce, pelo uso de cruzamentos dirigidos e pela terminação em confinamento. A adoção dessas inovações tecnológicas objetiva principalmente maior produção de carne de melhor qualidade em menos tempo e em menor área. Para melhor nortear as recomendações de raças e de esquemas de cruzamentos adequados aos sistemas de terminação que visam à produção do bovino jovem, são necessárias informações sobre a capacidade de consumo de alimentos e sobre a eficiência alimentar das raças e dos cruzamentos mais promissores para a região. Informações sobre a possibilidade de existência de heterose para as mencionadas características biológicas são igualmente relevantes.

Nesse contexto, o presente estudo teve como objetivo medir o consumo voluntário de alimentos e a conversão alimentar de bovinos machos inteiros das raças Charolês, Caracu e de seus cruzamentos recíprocos, quando confinados na mesma idade e durante o mesmo período de tempo.

\section{Material e Métodos}

Foram analisados o consumo diário de matéria seca (MS) por $100 \mathrm{~kg}$ de peso vivo (CPUPV), a conversão alimentar (CONV) e o ganho de peso médio diário em confinamento (GMD) de 80 machos bovinos inteiros, sendo 18 do grupo Charolês, 23 Charolês x Caracu, 20 Caracu x Charolês e 19 Caracu, nascidos na Estação Experimental Fazenda Modelo, do IAPAR, em Ponta Grossa-PR, nos anos de 1992/95. O grupo Charolês x Caracu incluía $3 \mathrm{~F} 1 \mathrm{~s}$, $93 / 4 \mathrm{Ch}+1 / 4 \mathrm{Ca}$ e $115 / 8 \mathrm{Ch}+3 / 8 \mathrm{Ca}$. Por outro lado, o grupo Caracu x Charolês incluía $2 \mathrm{~F} 1 \mathrm{~s}, 43 / 4 \mathrm{Ca}+1 / 4 \mathrm{Ch}$, $125 / 8 \mathrm{Ca}+3 / 8 \mathrm{Ch}$ e $211 / 16 \mathrm{Ca}+5 / 16 \mathrm{Ch}$. Esses grupos não foram desdobrados nas respectivas composições raciais porque tal procedimento resultaria em números insuficientes de observações por subclasse.

Mais detalhes sobre o plano de acasalamentos, bem como sobre o manejo pré-desmame desses animais, são fornecidos por PEROTTO et al. (1998). No período pós-desmame, os animais foram mantidos em pastagens de Hemarthria altissima, sendo suplementados durante o primeiro inverno (maio a agosto) com um concentrado (14,7\% de PB e $73 \%$ de NDT) fornecido à base de $1 \mathrm{~kg} /$ animal $\bullet$ dia.

Antes do início do segundo inverno pós-desmame e após terem sido submetidos a um período da adaptação ao confinamento de 14 dias, com peso inicial médio de $372 \mathrm{~kg}$ e idade média de 654 dias, os animais foram confinados em baias individuais por períodos de 76 a 97 dias, quando receberam silagem de milho à vontade mais uma ração concentrada (milho em grão moído, 69\%; soja moída, 20\%; farelo de soja, $10 \%$ e calcário, $1 \%$ ) fornecida à base de $1 \%$ do peso vivo do animal por dia, ajustado a cada pesagem realizada a intervalos de aproximadamente 28 dias, quando os animais foram submetidos previamente a um jejum por um período de 16 horas. Além da silagem e do concentrado, os animais receberam sal mineral e água à vontade. Diariamente, foram computadas as quantidades fornecidas e as sobras de silagem e concentrado. Submetido a análises laboratoriais, o concentrado forneceu a seguinte composição bromatológica: $88 \%$ MS, $17,8 \%$ PB, $0,45 \%$ Ca e $0,37 \%$ P. Por sua vez, a silagem de milho apresentou as seguintes características: $33,0 \%$ MS, 7,3\% PB e 3,4 pH.

Os dados das três características foram analisados pelo método dos quadrados mínimos (SAS, 1992), ajustando-se inicialmente um modelo linear que incluiu os efeitos fixos de grupo genético (GPO), ano do confinamento, do período (PRD) e das interações 
GPO*PRD, GPO*Ano e Ano*PRD, além da proporção de concentrado na MS consumida (RCV) como covariável. Nesta análise, foram considerados três períodos assim definidos: Período 1 (12/7 a 11/8/94, $30 / 3$ a $27 / 4 / 95,2 / 4$ a 30/4/96 e 26/5 a 23/6/97); Período 2 (12/8 a 8/9/94, 28/4 a 25/5/95, $1 / 5$ a 28/5/96 e 24/6 a 24/7/97); e Período 3 (9/9 a 26/9/94, 26/5 a 22/6/95, 29/5 a 8/7/96 e 25/7 a 18/8/97). Análises preliminares consideraram a inclusão do peso ao início do confinamento ou do peso médio durante o confinamento como covaráveis no modelo estatístico. Entretanto, essas covariáveis não foram mantidas nas análises definitivas, por se constatar que as mesmas influenciavam a magnitude do efeito de grupo genético.

$\mathrm{Na}$ segunda etapa, as variáveis foram analisadas para o período total, incluindo-se no modelo estatístico apenas efeitos com importância estatística $(\mathrm{P}<0,1)$. Finalmente, as características em estudo foram também analisadas por intermédio de um modelo de regressão múltipla (PEROTTO et al., 1998), em que o efeito fixo de grupo genético foi substituído por coeficientes representando os efeitos direto e materno da raça Charolês e os efeitos das heterozigoses individual e materna. Esta análise foi conduzida para se estimarem as médias para os "graus de sangue" representa- dos nos grupos Charolês x Caracu e Caracu x Charolês.

Estimativas de efeitos genéticos de interesse foram computadas como funções lineares dos efeitos de grupo genético e testadas estatisticamente pelo teste $t$.

\section{Resultados e Discussão}

Os resumos das análises de variância das três características analisadas considerando os efeitos do período são apresentados na Tabela 1. Os valores do $\mathrm{R}^{2}$ indicam que o modelo proposto descreveu adequadamente as diferenças entre animais para o CPUPV e para o GMD, mas não para a CONV, que apresentou alto coeficiente de variação $(35,92 \%)$. O grupo genético influenciou $(\mathrm{P}<0,01)$ o consumo e o ganho de peso e aproximou-se do nível de significância de $5 \%(\mathrm{P}<0,08)$ para a conversão alimentar. A proporção de concentrado na MS ingerida pelos animais teve efeito sobre o CPUPV $(\mathrm{P}<0,001)$, mas não sobre as outras duas características, embora, para a CONV, o efeito tenha mostrado alguma importância $(\mathrm{P}<0,10)$. Os efeitos de ano, período e interação Ano*PRD influenciaram as três variáveis analisadas. A interação GPO*PRD não mostrou efeito sobre qualquer das três características, enquanto a

Tabela 1 - Resumo das análises de variância do consumo diário de MS por $100 \mathrm{~kg}$ de peso vivo, da conversão alimentar e do ganho de peso médio diário de machos bovinos Charolês, Caracu e cruzamentos recíprocos em Ponta Grossa-PR, incluindo-se o período de alimentação no modelo estatístico

Table 1 - Summary of analyses of variance of daily DM intake per $100 \mathrm{~kg}$ of body weight, feed:gain ratio and average daily gain of Charolais, Caracu and reciprocal crosses in Ponta Grossa-PR, including the period of feeding in the statistical model

\begin{tabular}{|c|c|c|c|c|}
\hline \multirow[t]{2}{*}{$\begin{array}{l}\text { Fonte de variação } 1 / \text { Item } \\
\text { Source of variation }{ }^{1}\end{array}$} & \multirow[t]{2}{*}{$\begin{array}{l}\mathrm{gl} \\
d f\end{array}$} & \multicolumn{3}{|c|}{$\begin{array}{c}\text { Característica }^{2} / \text { Valor do } \mathrm{F} \\
\text { Trait }^{2} / \text { Computed } F \text { value }^{2}\end{array}$} \\
\hline & & CPUPV & $\mathrm{CONV}$ & GMD \\
\hline $\mathrm{RCV}$ & 1 & $533,90^{* * *}$ & 2,72 & 0,28 \\
\hline GPO & 3 & $5,91^{\text {*** }}$ & 2,06 & $6,81^{* * *}$ \\
\hline Ano/Year & 3 & $422,90^{* * *}$ & $5,88^{* * *}$ & $13,47^{* * * *}$ \\
\hline PRD & 2 & $85,65^{* * *}$ & $13,23^{* * *}$ & $6,88^{* *}$ \\
\hline GPO*PRD & 6 & 0,41 & 1,75 & 2,03 \\
\hline GPO*Ano & 9 & $2,24^{*}$ & 1,57 & 1,19 \\
\hline Ano*PRD & 6 & $46,60^{* * *}$ & $2,39^{*}$ & $9,42^{* * *}$ \\
\hline QM do Erro & & 0,00797 & 5,90715 & 0,11910 \\
\hline $\mathrm{R}^{2}$ & & 0,90 & 0,34 & 0,46 \\
\hline $\mathrm{CV}(\%)$ & & 3,96 & 35,92 & 21,65 \\
\hline Média/Mean & & 2,25 & 6,75 & 1,594 \\
\hline
\end{tabular}

$1 \mathrm{RCV}=$ Proporção de concentrado na MS, GPO = Grupo genético e PRD = Período, QM = Quadrado médio, $R^{2}$ = Coeficiente de determinação. ${ }^{2} \mathrm{CPUPV}=$ Consumo diário de MS por $100 \mathrm{~kg}$ de peso vivo animal (kg de MS/100 kg/dia), CONV = Conversão alimentar (kg de MS/kg de GMD) e GMD = Ganho médio diário em confinamento (kg/dia). ${ }^{* *}=\mathrm{P}<0,001,{ }^{* *}=\mathrm{P}<0,01,{ }^{*}=\mathrm{P}<0,05$.

$1 R C V=$ Proportion of concentrate in the $D M, G P O=$ Genetic group, $P R D=$ Period, $Q M=$ Mean square, $R^{2}=$ Coefficient of determination. ${ }^{2} \mathrm{CPUPV}=$ Daily DM intake per $100 \mathrm{~kg}$ of live animal weight ( $\mathrm{kg}$ of DM/100 kg BW/day), $C O N V=$ Feed :gain ratio $(\mathrm{kg}$ of $D M / \mathrm{kg}$ of $A D G)$ and $G M D=$ Average daily gain $(\mathrm{kg} /$ day $) .{ }^{* * *}=P<.001,{ }^{* *}=P<.01,{ }^{*}=P<.05$. 
interação GPO*Ano influenciou $(\mathrm{P}<0,05)$ o CPUPV, à exceção da CONV e do GMD.

Tendo em vista que as análises que incluíram os efeitos de período no modelo estatístico (Tabela 1) revelaram forte influência deste sobre qualquer das três variáveis, procurou-se examinar o comportamento das mesmas em cada um dos períodos considerados. Os resultados indicaram que o consumo diário de MS diminuiu do primeiro para o segundo período, mas aumentou do segundo para o terceiro. As médias para o CPUPV foram 2,22 $\pm 0,012,2,15$ $\pm 0,010$ e $2,39 \pm 0,014 \mathrm{~kg}$ de $\mathrm{MS} / 100 \mathrm{~kg}$ de $\mathrm{PV} / \mathrm{dia}$, respectivamente para os períodos 1,2 e 3 . Examinando-se o comportamento dos grupos genéticos quanto ao CPUPV, segundo o período (Tabela 2), observouse que o consumo diário de MS dos grupos $\mathrm{Ch}$ e $\mathrm{ChCa}$ se manteve estável do primeiro para o segundo período e aumentou do segundo para o terceiro período. Os grupos $\mathrm{Ca}$ e $\mathrm{CaCh}$ também consumiram mais MS no último período em relação ao segundo, porém o consumo durante o segundo período foi ligeiramente inferior $(\mathrm{P}<0,05)$ ao do primeiro. As médias para a conversão alimentar segundo o período foram $5,32 \pm 0,33,6,47 \pm 0,29$ e 8,32 $\pm 0,37 \mathrm{~kg}$ de $\mathrm{MS} / \mathrm{kg}$ de GMD, respectivamente para os períodos 1 , 2 e 3 , sendo estatisticamente significativas $(\mathrm{P}<0,01)$ qualquer das possíveis comparações entre períodos. Tais diferenças podem ser explicadas pela alteração na composição do ganho de peso, à medida que o estádio de maturidade avança (WEBSTER, 1989). Comportamento similar é observado quando a conversão de cada grupo genético é examinada em função do período (Tabela 3). Entretanto, em razão do aumento dos erros-padrão das médias, algumas das diferenças estatísticas entre períodos desaparecem. Assim, as conversões dos grupos $\mathrm{Ch}$ e $\mathrm{ChCa}$

Tabela 2 - Médias para o consumo diário de MS por $100 \mathrm{~kg}$ de peso vivo de machos bovinos Charolês, Caracu e cruzamentos recíprocos em Ponta Grossa-PR, incluindo-se o período de alimentação no modelo estatístico

Table 2 - Least squares means for daily DM intake per $100 \mathrm{~kg}$ of body weight of Charolais, Caracu and reciprocal crosses in Ponta Grossa-PR, including the period of feeding in the statistical model

\begin{tabular}{lccc}
\hline $\begin{array}{l}\text { Grupo genético } \\
\text { Genetic group }^{1}\end{array}$ & \multicolumn{3}{c}{$\begin{array}{c}\text { Período } \\
\text { Period }\end{array}$} \\
\cline { 2 - 4 } & 1 & 2 & 3 \\
\hline Charolês & $2,20 \pm 0,022^{\mathrm{a}}$ & $2,15 \pm 0,021^{\mathrm{a}}$ & $2,38 \pm 0,023^{\mathrm{b}}$ \\
ChCa & $2,18 \pm 0,019^{\mathrm{a}}$ & $2,13 \pm 0,019^{\mathrm{a}}$ & $2,35 \pm 0,021^{\mathrm{b}}$ \\
CaCh & $2,24 \pm 0,022^{\mathrm{a}}$ & $2,17 \pm 0,020^{\mathrm{b}}$ & $2,42 \pm 0,023^{\mathrm{c}}$ \\
Caracu & $2,24 \pm 0,022^{\mathrm{a}}$ & $2,15 \pm 0,021^{\mathrm{b}}$ & $2,42 \pm 0,022^{\mathrm{c}}$ \\
\hline
\end{tabular}

$1 \mathrm{ChCa}=$ Mestiço Charolês $\times$ Caracu filho de touro Charolês, $\mathrm{CaCh}=$ Mestiço Caracu $\times$ Charolês filho de touro Caracu. Médias na linha seguidas de letras diferentes são diferentes $(P<0,05)$ pelo teste t.

1 Charolês = Charolais, ChCa $=$ Crossbred Charolais $x$ Caracu sired by Charolais bull, CaCh $=$ Crossbred Caracu $x$ Charolais sired by Caracu bull. Means within a row followed by different letters are different $\left(P_{<.05)}\right.$ by $t$ test.

Tabela 3 - Médias para a conversão alimentar de machos bovinos Charolês, Caracu e cruzamentos recíprocos em Ponta Grossa-PR, incluindo-se o período de alimentação no modelo estatístico

Table 3 - Least squares means for feed:gain ratio of Charolais, Caracu and reciprocal crosses in Ponta Grossa-PR, including the period of feeding in the statistical model

\begin{tabular}{lccc}
\hline $\begin{array}{l}\text { Grupo genético } \\
\text { Genetic group }\end{array}$ & \multicolumn{3}{c}{$\begin{array}{c}\text { Período } \\
\text { Period }\end{array}$} \\
\cline { 2 - 4 } & 1 & 2 & 3 \\
\hline Charolês & $5,03 \pm 0,61^{\mathrm{a}}$ & $6,31 \pm 0,59^{\mathrm{ab}}$ & $7,60 \pm 0,62^{\mathrm{b}}$ \\
$\mathrm{ChCa}$ & $4,55 \pm 0,53^{\mathrm{a}}$ & $6,64 \pm 0,51^{\mathrm{b}}$ & $7,71 \pm 0,57^{\mathrm{b}}$ \\
$\mathrm{CaCh}$ & $5,24 \pm 0,59^{\mathrm{a}}$ & $6,44 \pm 0,56^{\mathrm{a}}$ & $9,78 \pm 0,62^{\mathrm{b}}$ \\
$\mathrm{Caracu}$ & $6,44 \pm 0,60^{\mathrm{a}}$ & $6,48 \pm 0,57^{\mathrm{a}}$ & $8,18 \pm 0,61^{\mathrm{b}}$ \\
\hline 1 ChCa = Mestiço Charolês $\times$ Caracu filho de touro Charolês, CaCh $=$ Mestiço Caracu $\times$ Charolês filho de \\
touro Caracu. Médias na linha seguidas de letras diferentes são diferentes $(\mathrm{P}<0,05)$ pelo teste t. \\
1 Charolês=Charolais, ChCa=CrossbredCharolais $x$ Caracusired by Charolais bull, CaCh=Crossbred Caracux Charolais \\
\multicolumn{2}{l}{ sired by Caracu bull. Means within a row followed by different letters are different $(P<.05)$ by $t$ test. }
\end{tabular}


não diferiram do segundo para o terceiro período. Ao contrário, os grupos $\mathrm{Ca}$ e $\mathrm{CaCh}$ apresentaram conversões piores no último período em comparação às dos períodos anteriores, que não diferiram entre si. Estudando a conversão alimentar de bovinos Nelore, 3/4 Nelore-Charolês, 3/4 Nelore-Fleckvieh e 3/4 Nelore-Chianina, em função do grau de maturidade, EUCLIDES FILHO et al. (1997) verificaram que a conversão apresentada pela raça Nelore aumentou de maneira constante com o progresso do grau de maturidade, ao passo que para os demais grupos a conversão se manteve estável do segundo para o terceiro período. Em concordância com o presente estudo, tais resultados evidenciam a necessidade de se definir o peso ideal de abate, em função do grupo genético, quando o objetivo for a obtenção de melhor eficiência de utilização de alimentos.

A Tabela 4 apresenta o comportamento do GMD segundo o período experimental. Observa-se que os ganhos do grupo $\mathrm{ChCa}$ reduziram drasticamente $(\mathrm{P}<0,001)$ do primeiro para o segundo período e se estabilizaram $(\mathrm{P}>0,05)$ do segundo para o terceiro período. O GMD do grupo $\mathrm{CaCh}$ não chegou a diferir do primeiro para o segundo período $(\mathrm{P}>0,05)$, porém houve diferença significativa $(\mathrm{P}<0,05)$ entre os GMDs do segundo e do terceiro período. Por outro lado, os GMDs dos grupos Charolês e Caracu não diferiram ( $\mathrm{P}>0,05)$ com o período.

Como a interação GPO*PRD não mostrou influência significativa sobre as características em estudo, essas foram analisadas para o período total, considerando-se apenas os efeitos de grupo genético, ano do confinamento e proporção de concentrado na MS ingerida. Note-se que a eliminação de termos supérfluos do modelo estatístico não comprometeu a qualidade das análises, pois os valores dos coeficientes de determinação estatística $\left(\mathrm{R}^{2}\right)$ mantiveram-se praticamente inalterados da Tabela 1 para a Tabela 5 .

As médias dos quatro grupos genéticos, obtidas pelo método dos quadrados mínimos, para as três características, considerando-se o período total, são apresentadas na Tabela 6. Resultados de contrastes de interesse entre médias ou entre conjuntos de médias são mostrados na Tabela 7.

O consumo diário de MS por $100 \mathrm{~kg}$ de peso vivo apresentou média global de 2,25 kg. Este valor é ligeiramente inferior aos $2,34 \mathrm{~kg}$ reportados por RESTLE et al. (1995) para a raça Charolês e bastante inferior aos $2,71 \mathrm{~kg}$ encontrados por CASACCIA et al. (1993) para a mesma raça. Consumo diário de MS por $100 \mathrm{~kg}$ de peso vivo da ordem de $2,55 \mathrm{~kg}$ é apresentado também por EUCLIDES FILHO et al. (1997) para machos inteiros 3/4Nelore+1/4Charolês. As discordâncias entre os valores de consumo encontrados nesses estudos poderiam ser atribuídas a diferenças nas dietas, na idade, no peso e no nível genético dos animais utilizados nos experimentos. No presente caso, a relação concentrado:volumoso na MS foi 0,33:0,67.

$\mathrm{O}$ efeito genético aditivo (Ch-Ca) e o efeito heterótico (Tabela 7) não foram significativos para a CPUPV. Da mesma forma, não foi significativa a diferença entre a média dos cruzamentos recíprocos e a média do Charolês. Esta diferença tem interesse, porque expressa o desempenho do esquema alternado de cruzamentos como desvio a partir do Charolês, a raça paterna superior. Por outro lado, a diferença recíproca $(\mathrm{ChCa}-\mathrm{CaCh})$ foi significativa $(\mathrm{P}<0,05)$, sendo que mestiços com maior predominância de

Tabela 4 - Médias para ganho de peso médio diário de machos bovinos Charolês, Caracu e cruzamentos recíprocos em Ponta Grossa-PR, incluindo-se o período de alimentação no modelo estatístico

Table 4 - Least squares means for average daily gain of Charolais, Caracu and reciprocal crosses in Ponta Grossa-PR, including the period of feeding in the statistical model

\begin{tabular}{lccc}
\hline $\begin{array}{l}\text { Grupo genético } \\
\text { Genetic group }\end{array}$ & \multicolumn{3}{c}{$\begin{array}{c}\text { Período } \\
\text { Period }\end{array}$} \\
\cline { 2 - 4 } & 1 & 2 & 3 \\
\hline Charolês & $1,717 \pm 0,086^{\mathrm{a}}$ & $1,546 \pm 0,083^{\mathrm{a}}$ & $1,584 \pm 0,088^{\mathrm{a}}$ \\
$\mathrm{ChCa}$ & $2,033 \pm 0,075^{\mathrm{a}}$ & $1,586 \pm 0,072^{\mathrm{b}}$ & $1,611 \pm 0,081^{\mathrm{b}}$ \\
$\mathrm{CaCh}$ & $1,723 \pm 0,084^{\mathrm{a}}$ & $1,541 \pm 0,080^{\mathrm{a}}$ & $1,395 \pm 0,088^{\mathrm{b}}$ \\
$\mathrm{Caracu}$ & $1,489 \pm 0,085^{\mathrm{a}}$ & $1,487 \pm 0,081^{\mathrm{a}}$ & $1,437 \pm 0,087^{\mathrm{a}}$ \\
\hline 1 ChCa = Mestiço Charolês $\times$ Caracu filho de touro Charolês, CaCh $=$ Mestiço Caracu $\times$ Charolês filho de \\
touro Caracu. Médias na linha seguidas de letras diferentes são diferentes $(\mathrm{P}<0,05)$ pelo teste t. \\
1 Charolês = Charolais, ChCa = Crossbred Charolais $\times$ Caracu sired by Charolais bull, CaCh $=$ Crossbred Caracu $x$ \\
\multicolumn{2}{c}{ Charolais sired by Caracu bull. Means within a row followed by different letters are different $(P<$. O5) by $t$ test. }
\end{tabular}


Tabela 5 - Resumo das análises de variância do consumo diário de MS por $100 \mathrm{~kg}$ de peso vivo, da conversão alimentar e do ganho de peso médio diário de machos bovinos Charolês, Caracu e cruzamentos recíprocos em Ponta Grossa-PR, no período total

Table 5 - Summary of analyses of variance of daily DM intake per $100 \mathrm{~kg}$ of body weight, feed:gain ratio and average daily gain of Charolais, Caracu and reciprocal crosses in Ponta Grossa-PR, for the total period

\begin{tabular}{|c|c|c|c|c|}
\hline \multirow[t]{2}{*}{$\begin{array}{l}\text { Fonte de variação }{ }^{1} / \text { Item } \\
\text { Source of variation }\end{array}$} & \multirow[t]{2}{*}{$\begin{array}{l}\mathrm{gl} \\
d f\end{array}$} & \multicolumn{3}{|c|}{$\begin{array}{l}\text { Característica }^{2} / \text { Valor do } \mathrm{F} \\
\text { Trait }^{2} / \text { Computed }^{F} \text { value }\end{array}$} \\
\hline & & CPUPV & CONV & GMD \\
\hline GPO & 3 & $3,36^{*}$ & $2,83^{*}$ & $6,35^{\text {*** }}$ \\
\hline Ano/Year & 3 & $286,41^{* * *}$ & $9,98^{* * *}$ & $3,10^{* * *}$ \\
\hline $\mathrm{RCV}$ & 1 & $406.46^{* * *}$ & $8,13^{* *}$ & $1,38^{\mathrm{NS}}$ \\
\hline QM do Erro & & 0,00396 & 1,62193 & 0,03020 \\
\hline $\mathrm{R}^{2}$ & & 0,92 & 0,36 & 0,47 \\
\hline $\mathrm{CV}(\%)$ & & 2,80 & 18,87 & 12,64 \\
\hline Média/Mean & & 2,25 & 6,75 & 1,587 \\
\hline
\end{tabular}

$1 \mathrm{GPO}=$ Grupo genético e RCV = Proporção de concentrado na MS. QM = Quadrado médio, $\mathrm{R}^{2}=$ Coeficiente de determinação. ${ }^{2} \mathrm{CPUPV}=$ Consumo diário de MS por $100 \mathrm{~kg}$ de peso vivo animal $(\mathrm{kg}$ de MS $/ 100 \mathrm{~kg} / \mathrm{dia}$ ), CONV = Conversão alimentar ( $\mathrm{kg}$ de MS $/ \mathrm{kg}$ de GMD) e GMD = Ganho médio diário em confinamento (kg/dia). ${ }^{* * *}=\mathrm{P}<0,001,{ }^{* *}=\mathrm{P}<0,01,{ }^{*}=\mathrm{P}<0,05$ e $\mathrm{NS}^{\mathrm{N}} \mathrm{P}>0,05$.

${ }_{1} G P O=$ Genetic group and RCV $=$ Proportion of concentrate in the $D M$. $Q M=$ Mean squares, $R^{2}=$ Coefficient of determination. ${ }^{2} C P U P V=$ Daily DM intake per $100 \mathrm{~kg}$ of live animal weight $(\mathrm{kg}$ of DM/100 kg/day), CONV = Feed:gain ratio $(\mathrm{kg}$ of $D M / \mathrm{kg}$ of $A D G)$ and $G M D=$ Average daily gain $\left(\mathrm{kg} /\right.$ day). ${ }^{* * *}=P<.001,{ }^{* *}=P<.01,{ }^{*}=P<.05$ and ${ }^{N S}=P>05$.

Tabela 6 - Médias para o consumo diário de MS por 100 kg de peso vivo, para a conversão alimentar e o ganho de peso médio diário de machos bovinos Charolês, Caracu e cruzamentos recíprocos em Ponta grossa-PR para o período total

Table 6 - Least squares means for daily DM intake per $100 \mathrm{~kg}$ of body weight, feed:gain ratio and average daily gain of Charolais, Caracu and reciprocal crosses in Ponta Grossa-PR, for the total period

\begin{tabular}{lccc}
\hline $\begin{array}{l}\text { Grupo genético } \\
\text { Genetic group }\end{array}$ & \multicolumn{3}{c}{$\begin{array}{c}\text { Característica }^{2} \\
\text { Trait }^{2}\end{array}$} \\
\cline { 2 - 4 } & CPUPV & CONV & GMD \\
\hline Charolês/Charolais & $2,24 \pm 0,015$ & $6,22 \pm 0,30$ & $1,619 \pm 0,047$ \\
ChCa & $2,22 \pm 0,013$ & $6,36 \pm 0,27$ & $1,722 \pm 0,042$ \\
CaCh & $2,28 \pm 0,014$ & $7,18 \pm 0,28$ & $1,552 \pm 0,045$ \\
Caracu & $2,26 \pm 0,015$ & $7,08 \pm 0,29$ & $1,456 \pm 0,046$ \\
\hline
\end{tabular}

$1 \mathrm{ChCa}=$ Mestiço Charolês $\times$ Caracu filho de touro Charolês, $\mathrm{CaCh}=$ Mestiço Caracu $\times$ Charolês filho de touro Caracu. ${ }^{2}$ CPUPV = Consumo diário de MS por $100 \mathrm{~kg}$ de peso vivo animal (kg de MS/100 $\mathrm{kg} / \mathrm{dia}$ ), CONV = Conversão alimentar ( $\mathrm{kg}$ de MS/kg de GMD) e GMD = Ganho médio diário em confinamento $(\mathrm{kg} / \mathrm{dia})$.

$1 \mathrm{ChCa}=$ Crossbred Charolais $x$ Caracu sired by Charolais bull, CaCh $=$ Crossbred Caracu $x$ Charolais sired by Caracu bull. ${ }^{2} C P U P V=$ Daily $D M$ intake per $100 \mathrm{~kg}$ of live animal weight $(\mathrm{kg}$ of DM $/ 100 \mathrm{~kg} \mathrm{BW} /$ day $), C O N V=$ Feed:gain ratio ( $\mathrm{kg}$ of $D M / \mathrm{kg}$ of $A D G)$ and $G M D=$ Average daily gain $(\mathrm{kg} /$ day).

germoplasma Caracu ingeriram mais MS que seus recíprocos. Note-se que, embora as diferenças não tenham sido significativas, os maiores consumos diários de MS sempre corresponderam aos grupos com maior predominância de germoplasma Caracu, a raça mais rústica.

As médias para a conversão alimentar variaram de $6,22 \pm 0,30 \mathrm{~kg}$ de MS/kg de GMD para o grupo Charolês a 7,18 $\pm 0,28 \mathrm{~kg}$ de MS/kg de GMD para o $\mathrm{CaCh}$. O valor encontrado, nesta pesquisa, para o
Charolês é melhor que os 7,52 kg de MS/kg de GMD reportados por CASACCIA et al. (1993) para a mesma raça. Esses autores trabalharam com uma dieta em que o volumoso (64\% da MS) era representado por silagem de sorgo, cujo valor nutritivo geralmente é inferior ao da silagem de milho utilizada neste trabalho. Pela análise dos resultados da Tabela 7, podese inferir que as diferenças Ch-Ca $(-0,861 \pm 0,42 \mathrm{~kg}$ de $\mathrm{MS} / \mathrm{kg}$ de GMD) e ChCa-CaCh $(-0,820 \pm 0,39 \mathrm{~kg}$ de $\mathrm{MS} / \mathrm{kg}$ de GMD) foram significativas $(\mathrm{P}<0,05)$, indi- 
Tabela 7 - Estimativas de efeitos genéticos para consumo diário de MS por $100 \mathrm{~kg}$ de peso vivo, conversão alimentar e ganho de peso médio diário de machos bovinos Charolês, Caracu e cruzamentos recíprocos em Ponta Grossa-PR para o período total

Table 7 - Estimates of genetic effects for daily DM intake per $100 \mathrm{~kg}$ of body weight, feed:gain ratio and average daily gain of Charolais, Caracu and reciprocal crosses in Ponta Grossa-PR, for the total period

Efeito genético $^{1}$

Genetic effect ${ }^{1}$

\begin{tabular}{lccl}
\cline { 2 - 4 } & \multicolumn{1}{c}{ CPUPV } & CONV & GMD \\
\hline Ch - Ca & $-0,026 \pm 0,02$ & $-0,861 \pm 0,42^{*}$ & $0,163 \pm 0,06^{* *}$ \\
Heterose & $-0,004 \pm 0,01$ & $0,114 \pm 0,29$ & $0,100 \pm 0,04^{*}$ \\
(ChCa+CaCh)/2-Ch & $0,009 \pm 0,02$ & $0,545 \pm 0,36$ & $0,018 \pm 0,05$ \\
$\mathrm{ChCa}-\mathrm{CaCh}$ & $-0,056 \pm 0,02^{* *}$ & $-0,820 \pm 0,39^{*}$ & $0,170 \pm 0,06^{* *}$ \\
\hline
\end{tabular}

$1 \mathrm{Ch}=$ Charolês, $\mathrm{Ca}=$ Caracu, $\mathrm{ChCa}=$ Mestiço Charolês $\times$ Caracu filho de touro Charolês, $\mathrm{CaCh}=$ Mestiço Caracu $\mathrm{x}$ Charolês filho de touro Caracu. Heterose $=(\mathrm{ChCa}+\mathrm{CaCh}) / 2-(\mathrm{Ch}+\mathrm{Ca}) / 2 .{ }^{2} \mathrm{CPUPV}=$ Consumo diário de MS por 100 $\mathrm{kg}$ de peso vivo animal ( $\mathrm{kg}$ de MS $/ 100 \mathrm{~kg} / \mathrm{dia}$ ), CONV = Conversão alimentar (kg de MS/kg de GMD) e GMD = Ganho médio diário em confinamento (kg/dia). ${ }^{*}=\mathrm{P}<0,05 \mathrm{e}^{* *}=\mathrm{P}<0,01$ pelo teste t.

$1 \mathrm{ChCa}=$ Crossbred Charolais $x$ Caracu sired by Charolais bull, $\mathrm{CaCh}=$ Crossbred Caracu $x$ Charolais sired by Caracu bull. Heterosis $=(\mathrm{ChCa}+\mathrm{CaCh}) / 2-(\mathrm{Ch}+\mathrm{Ca}) / 2 .{ }^{2} \mathrm{CPUPV}=$ Daily DM intake per $100 \mathrm{~kg}$ of live animal weight $(\mathrm{kg}$ of DM $/ 100 \mathrm{~kg}$ BW/day $), C O N V=F e e d$ :gain ratio $(\mathrm{kg}$ of $D M / \mathrm{kg}$ of $A D G)$ and $G M D=$ Average daily gain $(\mathrm{kg} /$ day $) .{ }^{*}=P<.05$ and ${ }^{* *}=P<.01$ by $t$ test. cando que animais filhos de touros Charolês apresentaram melhor conversão alimentar que os filhos de touros Caracu. Ao se concluir sobre a existência de diferenças entre grupos genéticos quanto à conversão alimentar, deve-se considerar se as mesmas foram estabelecidas para determinado intervalo de tempo (idade constante), até determinado peso ou determinado estádio de maturidade. Os resultados encontrados, neste trabalho, são consistentes com as conclusões de MASON (1971) e WARWICK e COBB (1975), demonstrando que, quando se comparam animais da mesma faixa etária ou até determinado peso, são mais eficientes aqueles que têm maior velocidade de crescimento e menos gordura na carcaça ao término do confinamento. No presente estudo, as diferenças de conversão alimentar entre grupos podem ser atribuídas a diferenças na composição do ganho de peso. Esta afirmação tem suporte nos dados de composição da carcaça de machos inteiros Charolês, Caracu e cruzamentos recíprocos terminados em confinamento (MOLETTA et al., 1993), que apresentaram valores médios para a espessura de gordura de cobertura da carcaça de $0,81 \mathrm{~mm}$ para o $\mathrm{Ch}, 1,53 \mathrm{~mm}$ para o Ca, 0,81 mm para o $\mathrm{ChCa}$ e $0,99 \mathrm{~mm}$ para o $\mathrm{CaCh}$.

Outra preocupação quando se observam diferenças de eficiência alimentar entre grupos genéticos é verificar se as mesmas não estão refletindo apenas diferenças de estádios de maturidade. No presente estudo, os valores médios das relações entre o peso dos animais ao término do confinamento e a média dos pesos adultos das vacas dos correspondentes grupos genéticos (estimados como sendo o valor assintótico da função de Richards, RICHARDS, 1959) ajustada à seqüência de pares de observações pesoidade de cada vaca) foram similares $(1,02 ; 1,06 ; 1,04$; e 1,02, respectivamente, para o Charolês, $\mathrm{ChCa}$, $\mathrm{CaCh}$ e Caracu), permitindo afirmar que esses quatro grupos genéticos se enquadram no mesmo tipo biológico (raças européias de grande porte) e inferir que as diferenças de conversão não refletem diferenças de graus de maturidade. Apesar disso, os resultados de conversão alimentar relatados neste trabalho, vistos em conjunto com aqueles do referido estudo de composição de carcaça, indicam que o peso ideal de abate desses grupos deveria elevar-se com o aumento da proporção de Charolês no germoplasma do animal. Igualmente, estes resultados lançam dúvidas sobre o uso da relação entre o peso de abate dos machos e o peso adulto das vacas como critério para definir o estádio de maturidade.

As médias para o GMD variaram de $1,456 \mathrm{~kg} / \mathrm{d}$ para o Caracu a $1,722 \mathrm{~kg} / \mathrm{d}$ para o ChCa. Valores dessa ordem são reportados por CRUZ et al. (1996) para cruzamentos de Blonde D'Aquitaine, Canchim, Limousin e Piemontês com Nelore confinados dos 286 aos $480 \mathrm{~kg}$ de peso vivo, consumindo aproximadamente $2,42 \mathrm{~kg}$ de MS/100 de PV/d de uma dieta com $13 \%$ de PB e $70 \%$ de NDT. No presente estudo, o consumo diário de MS situou-se abaixo dos valores reportados na literatura (RESTLE et al., 1995; CASACCIA et al., 1993; e EUCLIDES FILHO et al. 1997), permitindo inferir que uma dieta capaz de 
proporcionar maior ingestão de MS poderia exacerbar ainda mais o potencial de ganho de peso de animais de grupos genéticos como os que participaram do presente trabalho. Além das diferenças $\mathrm{Ch}-\mathrm{Ca}$ e $\mathrm{ChCa}-$ $\mathrm{CaCh}$, a heterose $((\mathrm{ChCa}+\mathrm{CaCh}) / 2-(\mathrm{Ch}+\mathrm{Ca}) / 2)$ também revelou-se significativa para o GMD. O Ch superou o Caracu em $0,163 \pm 0,06 \mathrm{~kg} / \mathrm{dia}(\mathrm{P}<0,01)$ e o ChCa, o CaCh em $0,170 \pm 0,06 \mathrm{~kg} / \mathrm{dia}(\mathrm{P}<0,01)$. O efeito da heterose sobre o GMD foi de $0,100 \pm 0,04 \mathrm{~kg}$ / dia $(\mathrm{P}<0,05)$. A diferença entre a média dos cruzamentos recíprocos e a média do Charolês não alcançou significância estatística para o GMD.

Os resultados obtidos pela análise que empregou o modelo de regressão múltipla são apresentados na Tabela 8. As estimativas dos contrastes entre médias e as predições das médias para as várias composições raciais foram obtidas como funções lineares dos efeitos aditivo $\left(\mathrm{Ch}^{\mathrm{I}}\right)$, materno $\left(\mathrm{Ch}^{\mathrm{M}}\right)$, heterozigótico individual $\left(\mathrm{ChCa}^{\mathrm{I}}\right)$ e heterozigótico materno $\left(\mathrm{ChCa}^{\mathrm{M}}\right)$ incluídos no modelo estatístico. As estimativas desses efeitos foram, respectivamente: $-0,097 \pm 0,030$ $(\mathrm{P}<0,01), 0,072 \pm 0,028 \quad(\mathrm{P}<0,05),-0,018 \pm 0,025 \mathrm{e}$
0,014 $\pm 0,025$ para o CPUPV; $-1,698 \pm 0,602(\mathrm{P}<0,01)$, $0,804 \pm 0,559,-0,582 \pm 0,503$ e $0,893 \pm 0,504$ para a CONV; e, 0,351 $\pm 0,096(\mathrm{P}<0,001),-0,204 \pm 0,089$ $(\mathrm{P}<0,05), 0,142 \pm 0,081(\mathrm{P}<0,10)$ e $-0,002 \pm 0,081$ para o GMD. A estimativa da heterose retida nas gerações avançadas do cruzamento alternado entre Charolês e Caracu foi computada contrastando-se a média de uma população de mestiços recíprocos $((65 / 100 \mathrm{Ch}+35 /$ $100 \mathrm{Ca}+65 / 100 \mathrm{Ca}+35 / 100 \mathrm{Ch}) / 2$ ) com a média das raças paternas $((\mathrm{Ch}+\mathrm{Ca}) / 2)$. Esta população mestiça representa aproximadamente a média ponderada das várias composições raciais dos mestiços presentes no experimento. A eqüivalência entre a análise pela regressão múltipla e a anterior pode ser constatada comparando-se os valores da diferença $\mathrm{Ch}-\mathrm{Ca}$ das Tabelas 7 e 8 . Existe também correspondência entre a heterose da Tabela 7 e a heterose retida da Tabela 8, assim como entre os valores da diferença $\mathrm{ChCa}-\mathrm{CaCh}$ das duas Tabelas. Como os efeitos heterozigóticos $\left(\mathrm{ChCa}^{\mathrm{I}}\right.$ e $\left.\mathrm{ChCa}^{\mathrm{M}}\right)$ não se mostraram significativos para o CPUPV, nem para a CONV, as médias dessas características para as várias compo-

Tabela 8 - Efeitos genéticos e médias segundo a composição racial para consumo diário de MS por 100 kg de peso vivo, conversão alimentar e ganho de peso médio diário de machos bovinos Charolês, Caracu e cruzamentos recíprocos em Ponta Grossa-PR, para o período total, obtidas pelo método da regressão múltipla

Table 8 - Genetic effects and means for daily DM intake per $100 \mathrm{~kg}$ of body weight, feed:gain ratio and average daily gain of Charolais, Caracu and reciprocal crosses in Ponta Grossa-PR, for the total period, obtained by a multiple regression method

\begin{tabular}{|c|c|c|c|}
\hline \multirow[t]{2}{*}{$\begin{array}{l}\text { Efeito genético }{ }^{1} \\
\text { Genetic effect }\end{array}$} & \multicolumn{3}{|c|}{$\begin{array}{c}\text { Característica }^{2} \\
\text { Trait }^{2}\end{array}$} \\
\hline & CPUPV & CONV & GMD \\
\hline $\mathrm{Ch}-\mathrm{Ca}$ & $-0,025 \pm 0,020$ & $-0,895 \pm 0,406^{*}$ & $0,147 \pm 0,065^{*}$ \\
\hline Heterose do F1(F1 Heterosis) & $-0,018 \pm 0,025$ & $-0,582 \pm 0,503$ & $0,142 \pm 0,081$ \\
\hline Heterose retida (Retained heterosis) & $-0,003 \pm 0,014$ & $0,211 \pm 0,282$ & $0,092 \pm 0,045^{*}$ \\
\hline $\mathrm{ChCa}-\mathrm{CaCh}$ & $-0,072 \pm 0,028^{*}$ & $-0,804 \pm 0,559$ & $0,204 \pm 0,089^{*}$ \\
\hline \multicolumn{4}{|l|}{ Média (Mean) } \\
\hline Charolês (Charolais) (Ch) & $2,27 \pm 0,015$ & $6,27 \pm 0,294$ & $1,606 \pm 0,047$ \\
\hline Caraca $(\mathrm{Ca})$ & $2,30 \pm 0,014$ & $7,18 \pm 0,286$ & $1,458 \pm 0,046$ \\
\hline $1 / 2 \mathrm{Ch}+1 / 2 \mathrm{Ca}$ & $2,23 \pm 0,027$ & $5,75 \pm 0,545$ & $1,776 \pm 0,087$ \\
\hline $1 / 2 \mathrm{Ca}+1 / 2 \mathrm{Ch}$ & $2,30 \pm 0,027$ & $6,55 \pm 0,537$ & $1,572 \pm 0,086$ \\
\hline $3 / 4 \mathrm{Ch}+1 / 4 \mathrm{Ca}$ & $2,27 \pm 0,018$ & $6,91 \pm 0,359$ & $1,689 \pm 0,058$ \\
\hline $3 / 4 \mathrm{Ca}+1 / 4 \mathrm{Ch}$ & $2,31 \pm 0,019$ & $7,76 \pm 0,375$ & $1,514 \pm 0,060$ \\
\hline $5 / 8 \mathrm{Ch}+3 / 8 \mathrm{Ca}$ & $2,25 \pm 0,015$ & $6,33 \pm 0,289$ & $1,733 \pm 0,046$ \\
\hline $5 / 8 \mathrm{Ca}+3 / 8 \mathrm{Ch}$ & $2,31 \pm 0,015$ & $7,16 \pm 0,294$ & $1,543 \pm 0,047$ \\
\hline $11 / 16 \mathrm{Ch}+5 / 16 \mathrm{Ca}$ & $2,27 \pm 0,014$ & $6,62 \pm 0,272$ & $1,711 \pm 0,044$ \\
\hline $11 / 16 \mathrm{Ca}+5 / 16 \mathrm{Ch}$ & $2,31 \pm 0,014$ & $7,46 \pm 0,286$ & $1,528 \pm 0,046$ \\
\hline \multicolumn{4}{|c|}{ 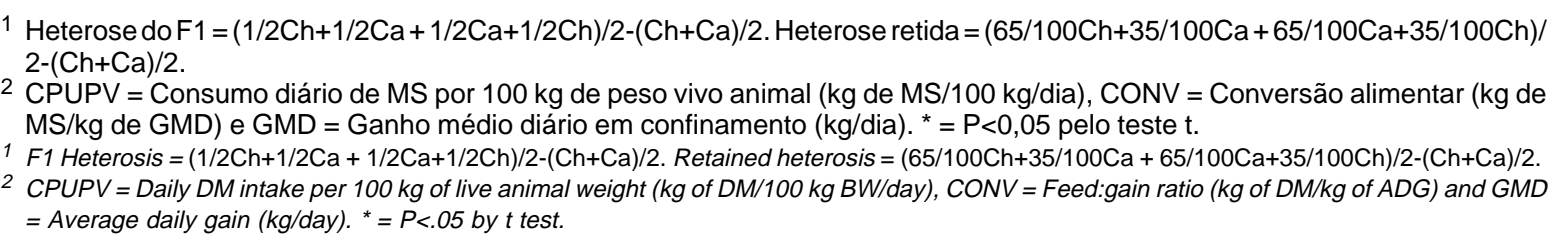 } \\
\hline
\end{tabular}


sições raciais são determinadas principalmente pelas proporções de Charolês e de Caracu nos genótipos dos indivíduos e de suas mães. Entretanto, para o GMD, o efeito da heterozigose do indivíduo mostrou alguma importância $(\mathrm{P}<0,01)$, resultado que se refletiu nas médias dessa característica e contribuiu para significância estatística da estimativa da heterose retida nas gerações avançadas do cruzamento alternado Charolês x Caracu. Note-se que, uma vez estimados os parâmetros do modelo de regressão múltipla, pode-se predizer o valor do desempenho de grupos genéticos não-contemplados no delineamento experimental, como é o caso do grupo $11 / 16 \mathrm{Ch}+5 /$ $16 \mathrm{Ca}$, que não estava presente no experimento cujos dados originaram o presente estudo.

\section{Conclusões}

Quando as comparações são restritas à mesma faixa etária ou até um peso pré-determinado, os animais Charolês ingerem a mesma quantidade de MS, porém apresentam melhor conversão alimentar que os Caracu.

Quando comparados em bases constantes de idade ou de peso, animais Charolês x Caracu ingerem menos MS e são mais eficientes quanto à conversão alimentar que seus recíprocos Caracu x Charolês.

A velocidade de ganho de peso de animais com maior proporção de germoplasma Charolês é superior àquela de animais com maior predominância de Caracu.

Os efeitos da heterose sobre o consumo de matéria seca e sobre a conversão alimentar foram insignificantes, mas houve manifestação de heterose para o ganho de peso médio diário em confinamento.

Com base nas três características analisadas, o desempenho de um sistema de cruzamentos alternados seria igual ao do Charolês e superior ao do Caracu.

\section{Referências Bibliográficas}

AGRICULTURAL RESEARCH SERVICE - USDA. 1974. Germplasm evaluation program progress report. ARS-NC13. Rep. № 1. U.S. Meat Animal Research Center, Clay Center, Nebr.

CASACCIA, J.L, PIRES, C.C, RESTLE, J. Confinamento de bovinos inteiros ou castrados de diferentes grupos genéticos. In: REUNIÃO ANUAL DA SOCIEDADE BRASILEIRA DE ZOOTECNIA, 30, 1993, Rio de Janeiro. Anais... Rio de Janeiro: SBZ, 1993, p.468.

CRUZ, G.M., TULIO, R.R., ESTEVES, S.N. et al. Desempenho em confinamento e características de carcaça de machos cruzados abatidos com diferentes pesos, para a produção do bovino jovem. In: REUNIÃO ANUAL DA SOCIEDADE BRASILEIRA DEZOOTECNIA, 33, 1996, Fortaleza. Anais...
Fortaleza: SBZ, 1996, p.203-205.

EUCLIDES FILHO, K., EUCLIDES, V.P.B., FIGUEIREDO, G.R. et al. 1997. Avaliação de animais Nelore e seus mestiços com Charolês, Fleckvieh e Chianina, em três dietas. 1. Ganho de peso e conversão alimentar. R. Bras. Zootec., 26(1):66-72.

GARRETT, W.N. 1980. Factors influencing energetic efficiency of beef production. J. Anim. Sci., 51(6):1434-1440.

GONÇALVES, L.C., SILVA, J.F.C., ESTEVÃO, M.M. 1991. Consumo e digestibilidade da matéria seca e da energia em zebuínos, e taurinos, seus mestiços e bubalinos. R. Soc. Bras. Zootec., 20(4):384-395.

LEDGER, H.P., ROGERSON, A., FREEMAN, G.H. 1970. Further studies on the voluntary food intake of Bos indicus, Bos taurus and crossbred cattle. Anim. Prod., 12(3):425-431.

LONG, C.R. 1980. Crossbreeding for beef production. J. Anim. Sci., 51(5):1197-11223.

MASON, I.L. Comparative beef performance of the large cattle breeds of Western Europe. 1971. Anim. Breed. Abstr., 39(1):1-29.

MOLETTA, J.L., PEROTTO, D., CUBAS, A.C. et al. Características de carcaça de bovinos Charolês, Caracu e cruzamentos recíprocos. In: REUNIÃO ANUAL DA SOCIEDADE BRASILEIRA DE ZOOTECNIA, 30, 1993, Rio de Janeiro. Anais... Rio de Janeiro: SBZ, 1993, p.260.

OLIVEIRA, M.A.T., FONTES, C.A.A., LANA, R.P. et al. 1994. Consumo alimentar e digestibilidade de rações com dois níveis de concentrado em bovinos de cinco grupos genéticos. R. Soc. Bras. Zootec., 23(4):667-677.

PEROTTO, D., MOLETTA, J.L., CUBAS, A.C. et al. 1998. Pesos ao nascimento e à desmama e ganho de peso do nascimento à desmama em bovinos das raças Charolesa e Caracu e cruzamentos recíprocos. R. Bras. Zootec., 27(4):730-737.

RESTLE, J., FELTEN, H.G., VAZ, F.N. Efeito da raça e heterose para desempenho em confinamento de novilhos de corte. In: REUNIÓN LATINO-AMERICANA DE PRODUCCIÓN ANIMAL, 14, 1995 Mar del Plata. Memorias... Balcarce:ALPA, 1995. p.852-854.

RICHARDS, J.F. 1959. A flexible growth function for empirical use. J. Exp. Bot., 10:290-300.

SAS. 1992. Technical Report P-229. SAS/STAT Software: Changes and Enhancements. Release 6.07. Cary: SAS Institute Inc. 620p.

WARWICK, E.J., COBB, E.H. 1975. Genetic variation in nutrition of cattle for meat production. In: THE EFFECT OF GENETIC VARIANCE ON NUTRITIONAL REQUIREMENTS OF ANIMALS. National Academy of Sciences. Washington, D.C. $123 \mathrm{p}$.

WEBSTER, A.J.F. 1989. Bioenergetics, bioengeneering and growth. Anim. Prod., 48(1):249-269.

Recebido em: 13/02/98

Aceito em: 03/08/99 